\title{
artigo
}

Godoi, J.; Campagnoli, M.; Fossa, A.M.; Rocha, M.C.P.; Lino, C.M.; Horibe, T.M.

Análise da demanda de pacientes com Doenças Crônicas Não Transmissiveis em uma Unidade de Pronto Atendimento;

\section{Análise da demanda de pacientes com Doenças Crônicas Não Transmissiveis em uma Unidade de Pronto Atendimento}

\author{
Analysis of the demand of patients with Chronic Non-Communicable Diseases in an Emergency Care Unit \\ Análisis de la demanda de pacientes con enfermedades crónicas no transmisibles en una unidad de atención de \\ emergencia
}

\begin{abstract}
RESUMO
Este estudo tem como objetivo analisar e caracterizar a demanda de pacientes com doença crônica não transmissível que utilizam a UPA, foram selecionadas as DCNT mais comuns entre a população de acordo com o Ministério da Saúde. Trata-se de um estudo descritivo, de natureza quantitativa, realizado em uma UPA, no município de Piracicaba, nos meses de maio e junho de 2018. Na coleta de dados foram analisados um total de 25.748 Fichas de Atendimento Ambulatorial, deste total foram selecionadas 582 fichas que apresentavam uma queixa relacionada a uma DCNT. Observou-se uma predominância do sexo feminino e a faixa etária entre 50 a 54 anos, a classificação de risco com maior frequência foi a da cor verde. As principais DCNT apresentadas foram lombalgia $(46,2 \% ; n=244)$ e asma $(21,6 \% ; n=114)$ e as queixas presentes no momento da triagem foram dor lombar $(54,4 \% ; n=287)$ e falta de $\operatorname{ar}(20,1 \% ; n=106)$, os pacientes desta UPA em sua maioria recebem alta após medicação ou alta com receita médica e o estudo evidência que há uma recidiva de usuários na unidade, o qual pode ser de 1 à 5 vezes no mesmo mês $(90,9 \% ; n=468)$. Conclui-se que houve pouco quantitativo de atendimentos relacionados a DCNT, as queixas mais comuns podem estar relacionadas ao clima do ano. Porém, algumas fichas não apresentavam concordância entre suas informações e as recidivas indicam que o usuário não procurou a atenção básica, que deve ser a principal porta de entrada para o sistema de saúde.
\end{abstract}

DESCRITORES: Unidade de Pronto Atendimento; Demanda de Pacientes; Doença Crônica Não Transmissível.

\section{ABSTRACT}

This study aims to analyze and characterize the demand of patients with chronic non-communicable disease using the UPA, the most common DCNTs were selected among the population according to the Ministry of Health. This is a descriptive study, of a quantitative nature, carried out in a UPA, in the city of Piracicaba, in the months of May and June 2018. In the data collection, a total of 25,748 Outpatient Care Files were analyzed, of this total 582 files were selected that presented a complaint related to an DCNT. There was a predominance of females and the age group between 50 to 54 years old, the risk classification with more frequency was the green color. The main DCNTs presented were low back pain $(46.2 \% ; n=244)$ and asthma $(21.6 \% ; n=114)$ and the complaints present at the time of screening were low back pain $(54.4 \% ; n=287)$ and shortness of breath $(20.1 \% ; n=106)$, most patients at this UPA are discharged after medication or discharged with a prescription and the study shows that there is a recurrence of users in the unit, which can be from 1 to 5 times in the same month $(90.9 \% ; n=468)$. It is concluded that there was little quantity of care related to DCNTs, the most common complaints may be related to the climate of the year. However, some forms did not show agreement between their information and the recurrences indicate that the user did not seek primary care, which should be the main gateway to the health system. DESCRIPTORS: Emergency Care Unit; Demand for Patients; Chronic Noncommunicable Disease.

\section{RESUMEN}

El objetivo de este estudio es analizar y caracterizar la demanda de pacientes con enfermedades crónicas no transmisibles utilizando la UPA, según el Ministerio de Salud, se seleccionaron las ENT más comunes entre la población. Se trata de un estudio descriptivo, de naturaleza cuantitativa, realizado en una UPA, en la ciudad de Piracicaba, en los meses de mayo y junio de 2018. En la recopilación de datos, se analizaron un total de 25,748 archivos de atención ambulatoria, de este total se seleccionaron 582 archivos que presentaron una queja relacionada con una ENT. Predominó el sexo femenino y el grupo de edad entre 50 y 54 años, la clasificación de riesgo con mayor frecuencia fue el color verde. Las principales ENT presentadas fueron dolor lumbar $(46,2 \% ; n=244)$ y asma $(21,6 \% ; n=114)$ y las quejas presentes en el momento del examen fueron dolor lumbar $(54,4 \% ; n=287)$ y dificultad para respirar $(20.1 \% ; n=106)$, la mayoría de los pacientes en este UPA son dados de alta después de la medicación o dados de alta con receta médica y el estudio muestra que hay una recurrencia de usuarios en la unidad, que puede ser de 1 a 5 veces en el mismo mes $(90.9 \% ; n=468)$. Se concluye que hubo poca cantidad de atención relacionada con las ENT, las quejas más comunes pueden estar relacionadas con el clima del año. Sin embargo, algunos formularios no mostraron acuerdo entre su información y las recurrencias indican que el usuario no buscó atención primaria, que debería ser la principal puerta de entrada al sistema de salud. DESCRIPTORES: Unidad de Atención de Emergencia; Demanda de Pacientes; Enfermedad Crónica No Transmisible.

RECEBIDO EM: 02/12/2019 APROVADO EM: 03/12/2019 


\section{Juliana Godoi}

Enfermeira. Universidade Metodista de Piracicaba, Piracicaba, SP, Brasil. https://orcid.org/0000-0002-4145-2332

\section{Michele Campagnoli}

Docente e Coordenadora Centro de Saúde, Mestre em Saúde Coletiva, Universidade Metodista de Piracicaba e Prefeitura Municipal de Campinas,Piracicaba, SP, Brasil. https://orcid.org/0000-0002-7874-2296

\section{Ângela Márcia Fossa}

Docente, Mestre em Saúde Coletiva, Universidade Metodista de Piracicaba, Piracicaba, SP, Brasi. https://orcid.org/00000001-5213-9694

\section{Maria Cristina Pauli da Rocha}

Coordenadora Graduação Enfermagem, Docente, Doutoranda Saúde da Criança Unicamp. Universidade Metodista de Piracicaba, Piracicaba, SP, Brasil. https://orcid.org/0000-0002-3563-6362

\section{Carolina Matteussi Lino}

Docente, Doutoranda em Saúde Coletiva, Universidade Metodista de Piracicaba, Piracicaba, SP, Brasil. https://orcid.org/00000001-6686-3296

\section{Tereza Mitsue Horibe}

Docente, Mestre em Saúde Coletiva, Universidade Metodista de Piracicaba, Piracicaba, SP, Brasil. https://orcid.org/00000002-7188-9287

\section{INTRODUÇÃO}

A $s$ redes de atenção à saúde (RAS) são arranjos organizativos de ações e serviços de saúde, integradas por meio de sistemas de apoio técnico, logístico e de gestão, nele temos diversos serviços interligados para uma melhor assistência à saúde ${ }^{(1)}$.

O primeiro contato do indivíduo nos serviços de saúde deve ser através da atenção básica $(\mathrm{AB})$, esta considerada a principal porta de entrada e centro de comunicação da $\operatorname{RAS}^{(2)}$.

Devido a estruturação das unidades de pronto atendimento (UPA), como funcionamento 24 horas, atendimento todos os dias da semana e diversificados tipos de assistências, percebe-se que a população entra pelo sistema de saúde através dela ${ }^{(3)}$.

Estudos $^{(4,5)}$ mostram que as procuras pelos atendimentos nas UPAs são feitas pelo usuário em busca de uma solução para seu problema de saúde, independente da gravidade. Isso é compreendido pela dificuldade do acesso aos serviços de $\mathrm{AB}$ e facilidade no atendimento emergencial, portanto é necessário que diversos serviços trabalhem juntos para qualificar a assistência.

Os usuários utilizam de forma indiscri- minada os serviços de pronto atendimento, apresentando queixas não urgentes e com atendimentos focados no problema atual, acreditam que o atendimento imediato com médicos e recursos de tecnologias duras, como exames laboratoriais e radiológicos, além de procedimentos de enfermagem, podem solucionar o caso ${ }^{(6)}$.

$\mathrm{Na}$ Inglaterra, criou-se a avaliação de risco em 1997, conhecida como Manchester, que serve para classificar os riscos de saúde do indivíduo, levando em conta a gravidade da queixa e determinando o tempo necessário de atendimento. Esse sistema foi implementado no Brasil em 2002, quando o Ministério da Saúde protocolou a Portaria n. ${ }^{\circ} 2.048$ para que todos os hospitais usassem uma classificação de risco para qualquer atendimento realizado nas unidades de pronto atendimento $^{(7)}$.

A subdivisão desta classificação corresponde entre vermelho ou nível 1 (emergente), onde o atendimento deve ser imediato; laranja ou nível 2 (muito urgente), quando a espera pode ser de até 10 minutos; amarela ou nível 3 (urgente), sendo até 60 minutos; verde ou nível 4 (pouco urgente), com até 120 minutos; e azul ou nível 5 (não urgente), podendo chegar a 240 minutos de espera ${ }^{(8)}$.
Existe uma necessidade da interação entre a atenção básica com as unidades de urgência e emergência para que as unidades básicas de saúde (UBS) e unidades de saúde da família (USF) realizem estratégias para acolher os usuários que apresentem casos de quadros agudos ou crônicos agudizados e que sejam compatíveis com suas limitações de funcionamento ${ }^{(9)}$.

Estes casos estão relacionados, em sua maioria, com doenças crônicas, que podem apresentar início gradual com longa e indefinida duração. Com o tempo, alguns casos crônicos podem apresentar mudanças, com períodos de agravamento dos sintomas, podendo estar associado com o envelhecimento populacional e o estilo de vida do paciente ${ }^{(3)}$.

Para combater o aumento e agravamento dos casos de DCNT, foi criado o Plano de Ação Estratégica para o Enfrentamento das Doenças Crônicas Não Transmissíveis no Brasil (2011-2022). Nele são destacados indicadores, metas, programas, projetos e atividades que diminuam o agravamento dessas doenças. Neste plano, o Ministério da Saúde destaca quatro grupos de DCNT, que são: as doenças circulatórias, câncer, doenças respiratórias crônicas e diabetes. Dentre esses grupos estão as doenças que apresen- 


\section{artigo}

Godoi, J.; Campagnoli, M.; Fossa, A.M.; Rocha, M.C.P.; Lino, C.M.; Horibe, T.M.;

Análise da demanda de pacientes com Doenças Crônicas Não Transmissiveis em uma Unidade de Pronto Atendimento;

tam maior incidência na população como a hipertensão arterial sistêmica (HAS), acidente vascular cerebral (AVC), câncer, asma, doença pulmonar obstrutiva crônica (DPOC), diabetes mellitus I e II ${ }^{(2)}$.

As faixas etárias que mais apresentam DCNT são adulto e/ou idoso, sendo mais prevalente em mulheres do que em homens ${ }^{(10)}$.

A lombalgia não está relacionada no Plano de Ação Estratégica para o Enfrentamento das DCNT pelo Ministério da Saúde, porém é uma doença crônicas com grande prevalência na população. As lombalgias podem ser caracterizadas por dores na região lombar inferior, lombosacrais ou sacroilíacas da coluna vertebral. A causa da dor pode estar relacionada com alterações musculoesqueléticas causadas pela má postura, excesso de peso, sedentarismo, entre outros e, geralmente, acomete indivíduos na faixa etária de $45 \operatorname{anos}^{(11)}$.

Portanto, este estudo pretende analisar a demanda dos atendimentos de pacientes com DCNT que procuram a unidade de pronto atendimento. Objetivou-se analisar o atendimento dos usuários com DCNT em uma Unidade de Pronto Atendimento. Como objetivos específicos: caracterizar qual DCNT é mais frequente na unidade; identificar o número de pacientes com DCNT que procuram a unidade; analisar a demanda por sexo e faixa etária; identificar o número de pacientes com mais de uma DCNT e caracterizar o desfecho do atendimento prestado.

\section{METODOLOGIA}

Trata-se de um estudo descritivo, de natureza quantitativa, esta é objetiva, centrada em dados e valores brutos, recorrendo à matemática para descrever seus resultados ${ }^{(12)}$. A pesquisa quantitativa é baseada no raciocínio lógico e dedutivo que possuam dados mensuráveis com validação numérica de uma determinada hipótese, utilizando dados estruturados e estatísticos $^{(13,14)}$.

Foi realizado em uma Unidade de
Pronto Atendimento no município de Piracicaba-SP. Os sujeitos da pesquisa foram fichas de atendimento de usuários que procuraram a unidade de pronto atendimento com relato de doenças crônicas não transmissíveis, como: diabetes Mellitus, hipertensão arterial sistêmica, acidente vascular cerebral, asma, DPOC e lombalgia. A pesquisa foi realizada durante os meses de maio e junho de 2018 .

Os critérios de inclusão estabelecidos foram: fichas de atendimento de usuários que se adequassem à faixa etária estabelecida de maiores ou iguais a 18 anos de idade (adulto e idoso), que apresentassem queixas de DCNT e que na hora do atendimento não estivessem em um estado agudizado. Os critérios de exclusão foram: fichas que não demonstrassem que o usuário possuía uma DCNT, que estivessem fora da faixa etária preconizada e que demonstrassem que a situação é agudizada. O câncer foi excluído da coleta, devido ao seu atendimento ser realizado em unidades especializadas.

A coleta de dados foi realizada através da avaliação das Fichas de Atendimento Ambulatorial (FAA) geradas na UPA, que identificassem relatos de DCNT e que estivessem dentro dos padrões de inclusão já citados.

O levantamento foi realizado através de uma planilha elaborada no Microsoft Excel com informações relevantes para análise das queixas apresentadas, como: quantidade de atendimentos dentro de 30 dias, idade, sexo, município, história da queixa, medicamentos em uso, sinais vitais, queixa principal e desfecho do atendimento.

Após a coleta dos dados nas FAAs, as informações foram transcritas em uma planilha feita no Microsoft Excel, e foram calculadas a frequência simples, média e porcentagem. Os dados foram apresentados em formas de gráficos e tabelas, relacionados com as literaturas atuais sobre o tema apresentado.

Os aspectos éticos foram respeitados em consonância com a Resolução do Conselho Nacional de Saúde n.o 466, de 2012, que regulamenta a realização de pesquisa em seres humanos. O protocolo de pesquisa foi submetido à apreciação ética e ao acompanhamento do Comitê de Ética em Pesquisa (CEP) da Universidade Metodista de Piracicaba (UNIMEP) obteve a aprovação através do protocolo n. ${ }^{\circ} 237.5290$ e foi solicitada a autorização da instituição, na qual os dados foram coletados. Somente após a aprovação pelo Comitê e autorização da instituição, a pesquisadora iniciou a coleta de dados.

As coletas foram feitas através das fichas de atendimento do paciente, portanto não houve contato com eles pessoalmente e nem por outras vias, como a telefônica ou digital. O trabalho manteve em anonimato e sigilo absoluto a identificação do sujeito em pesquisa, não contendo dados pessoais. No trabalho também não houve a identificação da unidade onde foram coletadas as informaçóes.

\section{RESULTADOS}

O município de Piracicaba tem os serviços subdivididos em diversas unidades, que englobam diversos níveis de atenção à saúde, como: os Programas de Saúde da Família, Unidades Básicas de Saúde, Centro de Referência para Atenção Básica e Consultório de Rua. Possui também clínicas especializadas, como: Centro de Atenção à Saúde do Adolescente, Centro de Doenças Infecto Contagiosas, Centro de Especialidades Odontológicas, Centro Especializado em Saúde da Mulher, Centro de Referência em Saúde do Trabalhador, Clínica de Atenção às Doenças Metabólicas, Clínica de Olhos, Policlínica e Centro de Atenção Psicossocial para portadores de doenças mentais, usuários de drogas e unidade infanto-juvenil. Também possui quatro Unidades de Pronto Atendimento Clínico e uma Central de Ortopedia e Traumatologia, além do Serviço de Atendimento Móvel de Urgência ${ }^{(15)}$.

O presente estudo foi realizado em uma UPA do município de Piracicaba, no período de maio a junho de 2018, e apresentou um total de 25.748 fichas de 


\section{Gráfico 1. Total de atendimentos de DCNT. Piracicaba, SP, Brasil, 2018.}

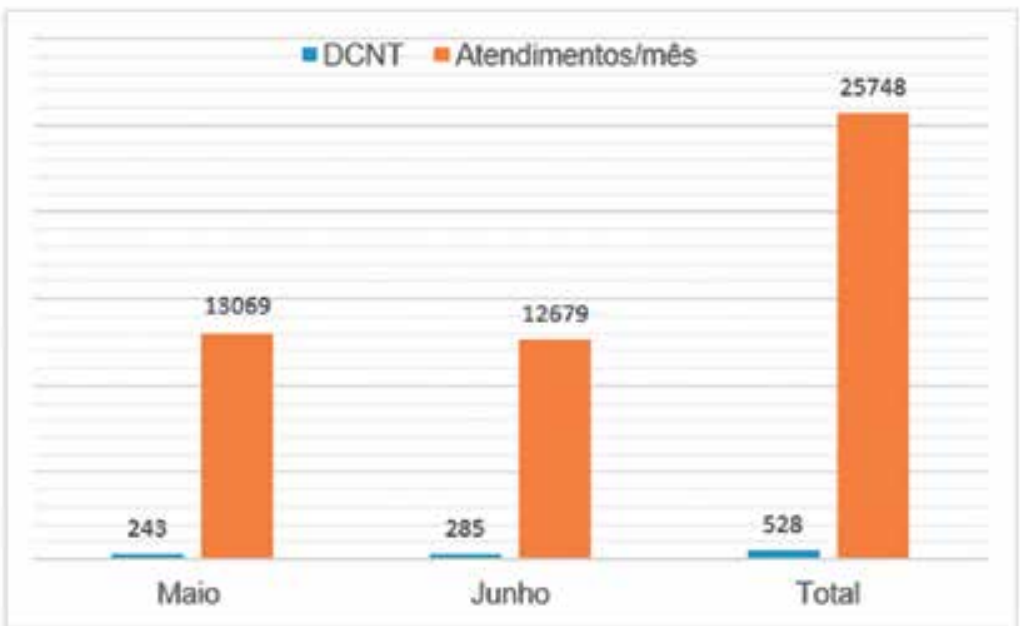

Tabela 1. Distribuição dos dados de acordo com o sexo. Piracicaba, SP, Brasil, 2018.

\begin{tabular}{lcccccc} 
& \multicolumn{2}{c}{ MAIO } & \multicolumn{2}{c}{ JUNHO } & \multicolumn{2}{c}{ TOTAL } \\
Sexo & No $^{\circ}$ & $\%$ & N $^{\circ}$ & $\%$ & $N^{\circ}$ & $\%$ \\
Feminino & 137 & $56,4 \%$ & 164 & $57,5 \%$ & 301 & $57 \%$ \\
Masculino & 106 & $43,6 \%$ & 121 & $42,5 \%$ & 227 & $43 \%$ \\
Total & 243 & $100 \%$ & 285 & $100 \%$ & 528 & $100 \%$
\end{tabular}

Tabela 2. Divisão dos atendimentos por faixa etária. Piracicaba, SP, Brasil, 2018.

\begin{tabular}{|c|c|c|c|c|c|c|}
\hline \multirow[b]{2}{*}{ Idade } & \multicolumn{2}{|c|}{ MAIO } & \multicolumn{2}{|c|}{ JUNHO } & \multicolumn{2}{|c|}{ TOTAL } \\
\hline & $N^{0}$ & $\%$ & $\mathrm{~N}^{0}$ & $\%$ & $\mathrm{~N}^{0}$ & $\%$ \\
\hline 15 a 19 anos & 1 & $0,4 \%$ & 5 & $1,75 \%$ & 6 & $1,1 \%$ \\
\hline 20 a 24 anos & 16 & $6,6 \%$ & 14 & $4,9 \%$ & 30 & $5,7 \%$ \\
\hline 25 a 29 anos & 20 & $8,2 \%$ & 22 & $7,7 \%$ & 42 & $7,9 \%$ \\
\hline 30 a 34 anos & 23 & $9,5 \%$ & 25 & $8,8 \%$ & 48 & $9,1 \%$ \\
\hline 35 a 39 anos & 20 & $8,2 \%$ & 22 & $7,7 \%$ & 42 & $7,9 \%$ \\
\hline 40 a 44 anos & 23 & $9,5 \%$ & 25 & $8,8 \%$ & 48 & $9,1 \%$ \\
\hline 45 a 49 anos & 24 & $9,9 \%$ & 24 & $8,4 \%$ & 48 & $9,1 \%$ \\
\hline 50 a 54 anos & 19 & $7,9 \%$ & 36 & $12,6 \%$ & 55 & $10,4 \%$ \\
\hline 55 a 59 anos & 14 & $5,8 \%$ & 26 & $9,1 \%$ & 40 & $7,6 \%$ \\
\hline 60 a 64 anos & 30 & $12,3 \%$ & 23 & $8,1 \%$ & 53 & $10,3 \%$ \\
\hline 65 a 69 anos & 18 & $7,4 \%$ & 18 & $6,3 \%$ & 36 & $6,8 \%$ \\
\hline 70 a 74 anos & 19 & $7,8 \%$ & 15 & $5,3 \%$ & 34 & $6,4 \%$ \\
\hline 75 a 79 anos & 7 & $2,9 \%$ & 14 & $4,9 \%$ & 21 & $3,9 \%$ \\
\hline 80 a 84 anos & 3 & $1,2 \%$ & 10 & $3,5 \%$ & 13 & $2,5 \%$ \\
\hline 85 a 89 anos & 4 & $1,6 \%$ & 4 & $1,4 \%$ & 8 & $1,5 \%$ \\
\hline 90 a 94 anos & 2 & $0,8 \%$ & 2 & $0,7 \%$ & 4 & $0,7 \%$ \\
\hline Total & 243 & $100 \%$ & 285 & $100 \%$ & 528 & $100 \%$ \\
\hline
\end{tabular}

atendimento. No Gráfico 1, observamos que em maio foram 13.069 atendimentos e em junho 12.679 atendimentos. Deste total, somente $2 \%(n=528)$ eram relacionadas a uma queixa de doenças crônicas, sendo $0,9 \%(\mathrm{n}=243)$ em maio e $1,1 \%$ $(n=285)$ em junho.

De acordo com a Tabela 1, observou-se a predominância do sexo feminino com $57 \%(\mathrm{n}=301)$.

$\mathrm{Na}$ Tabela 2, podemos identificar que o maior número de atendimentos foi de pacientes com a idade de 50 a 54 anos, sendo $10,4 \%(n=55)$ e as idades com menor demanda foi de 90 a 94 anos com $0,7 \%(n=4)$.

$\mathrm{O}$ usuário quando chega na UPA passa por uma triagem que classifica seu risco. Diante disso, podemos observar a predominância da classificação verde com 89,20\% ( $n=471)$, como mostra o Gráfico 2. Ressalva que a cor azul e vermelha não se aplicam ao levantamento dos dados realizado, já que a classificação azul não é utilizada na UPA e a cor vermelha o paciente entra, geralmente, pela emergência.

As DCNT descritas no trabalho foram divididas em dois subgrupos, os que possuem apenas uma patologia e os que possuem duas ou mais patologias associadas. O maior índice, como mostra a Tabela 3, foi de pessoas que apresentam lombalgia sem outra patologia de base com 46,2\% $(n=244)$. A lombalgia não é classificada pelo Ministério da Saúde como uma DCNT, porém, a procura por atendimento referente a esta causa é significativa, além disso segundo Lizier e colaboradores $^{(16)}$ de $15 \%$ a $20 \%$ dos adultos apresentam essa queixa.

O Gráfico 3 expõe uma maior incidência das queixas de lombalgia, apresentando $54,4 \%(\mathrm{n}=287)$ e falta de ar com $20,1 \%$ $(\mathrm{n}=106)$, que está relacionada à asma, este justificado pelo período em que o estudo foi realizado, sendo a estação fria do ano, o que contribui para o desencadeamento desta enfermidade.

A Tabela 4 mostra a relação entre as queixas, sinais vitais (SSVV) e as medicações prescritas, identificando quais apresentam concordância entre as queixas e o decorrer do atendimento. Percebe-se 


\section{artigo}

Godoi, J.; Campagnoli, M.; Fossa, A.M.; Rocha, M.C.P.; Lino, C.M.; Horibe, T.M.;

Análise da demanda de pacientes com Doenças Crônicas Não Transmissiveis em uma Unidade de Pronto Atendimento;

que as queixas apresentadas são, realmente, o motivo do atendimento com $69,9 \%$

$(\mathrm{n}=369)$, e as queixas não atendidas chegam a $15 \%(\mathrm{n}=81)$. Além disso, existe uma

Gráfico 2. Distribuição de dados de acordo com a classificação de risco. Piracicaba, SP, Brasil, 2018.

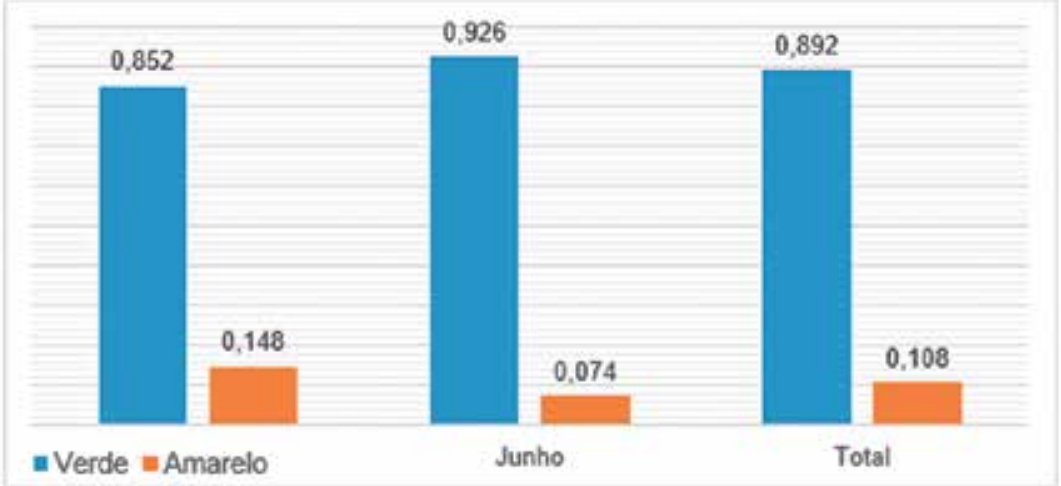

Tabela 3. Distribuição das DCNT. Piracicaba, SP, Brasil, 2018.

\begin{tabular}{|c|c|c|c|c|c|c|}
\hline \multirow[b]{2}{*}{ DCNT } & \multicolumn{2}{|c|}{ MAIO } & \multicolumn{2}{|c|}{ JUNHO } & \multicolumn{2}{|c|}{ TOTAL } \\
\hline & $\mathrm{N}^{\circ}$ & $\%$ & $\mathrm{~N}^{\circ}$ & $\%$ & $\mathrm{~N}^{\circ}$ & $\%$ \\
\hline HAS + AVC & 0 & 0 & 1 & $0,3 \%$ & 1 & $0,2 \%$ \\
\hline $\begin{array}{l}\text { HAS + DPOC + } \\
\text { Asma }\end{array}$ & 1 & $0,4 \%$ & 0 & 0 & 1 & $0,2 \%$ \\
\hline $\mathrm{DPOC}+$ Asma & 0 & 0 & 1 & $0,3 \%$ & 1 & $0,2 \%$ \\
\hline $\mathrm{DM}+\mathrm{ASMA}$ & 3 & $1,2 \%$ & 1 & $0,3 \%$ & 4 & $0,7 \%$ \\
\hline HAS + DPOC & 0 & 0 & 3 & $1,1 \%$ & 3 & $0,6 \%$ \\
\hline $\begin{array}{l}\text { HAS + DM + } \\
\text { Asma }\end{array}$ & 2 & $0,8 \%$ & 1 & $0,3 \%$ & 3 & $0,6 \%$ \\
\hline HAS + Asma & 3 & $1,2 \%$ & 4 & $1,4 \%$ & 7 & $1,3 \%$ \\
\hline DPOC & 11 & $4,5 \%$ & 1 & $0,3 \%$ & 12 & $2,3 \%$ \\
\hline $\mathrm{DM}$ & 6 & $2,5 \%$ & 9 & $3,2 \%$ & 15 & $2,8 \%$ \\
\hline HAS + DM & 14 & $5,8 \%$ & 14 & $4,9 \%$ & 28 & $5,3 \%$ \\
\hline HAS & 48 & $19,8 \%$ & 47 & $16,5 \%$ & 95 & $18 \%$ \\
\hline Asma & 48 & $19,8 \%$ & 66 & $23,2 \%$ & 114 & $21,6 \%$ \\
\hline Lombalgia & 107 & $44 \%$ & 137 & $48,1 \%$ & 244 & $46,2 \%$ \\
\hline Total & 243 & $100 \%$ & 285 & $100 \%$ & 528 & $100 \%$ \\
\hline 85 a 89 anos & 4 & $1,6 \%$ & 4 & $1,4 \%$ & 8 & $1,5 \%$ \\
\hline 90 a 94 anos & 2 & $0,8 \%$ & 2 & $0,7 \%$ & 4 & $0,7 \%$ \\
\hline Total & 243 & $100 \%$ & 285 & $100 \%$ & 528 & $100 \%$ \\
\hline
\end{tabular}

diferença da medicação com a queixa, o que equivale $1,9 \%(\mathrm{n}=10)$.

A maior prevalência nos desfechos dos atendimentos é a alta após a medicação com 83,1 (n=399) - Gráfico 4.

$\mathrm{Na}$ Tabela 5, podemos observar a recidiva dos usuários na unidade dentro de 30 dias, sendo por queixas distintas ou pela mesma sintomatologia. Neste caso, houve uma predominância nas repetições de atendimentos de 1 a 5 vezes no mês, o que representa 90,9\% $(\mathrm{n}=468)$ dos atendimentos. No mês de maio, 13 fichas não apresentavam essa informação devido ao fato de serem feitas manualmente, a unidade estava sem sistema neste período, portanto não foram utilizadas nesta análise.

Com os resultados, podemos observar que os valores dos atendimentos realizados na unidade são elevados, porém, os dados de DCNT não são significativos como esperado no início do trabalho. Houve a predominância do sexo feminino, a idade de 50 a 54 anos e, em sua maioria, com uma patologia de base. Grande parte dos atendimentos obtive a classificação verde, os quais poderiam ter sido encaminhados para a atenção básica, após o atendimento médico.

Em relação ao atendimento prestado no local, percebe-se que, em alguns momentos, ocorre uma discordância entre as queixas e os cuidados prestados. A maioria dos pacientes é liberada sem encaminhamento a outros serviços, o que pode ser a causa de uma nova procura à UPA.

\section{DISCUSSÃO}

$\mathrm{Na}$ pesquisa realizada houve mais atendimentos a pessoas do sexo feminino (57\%), este dado se assemelha a outros estudos, como de Tomberg e colaboradores $^{(17)}$ com $50,7 \%$, este teve o objetivo de analisar a demanda de atendimentos de acordo com a queixa e, Ribeiro e colaboradores ${ }^{(18)}$, com $53,6 \%$, que teve o objetivo de demarcar a demanda de atendimentos de acordo com a faixa etária. Os autores associam esta demanda a uma preocupação maior da mulher com o processo de saúde-doença.

Este dado é confirmado com os dados 
Gráfico 3. Distribuição dos dados de acordo com as queixas. Piracicaba, SP, Brasil, 2018.

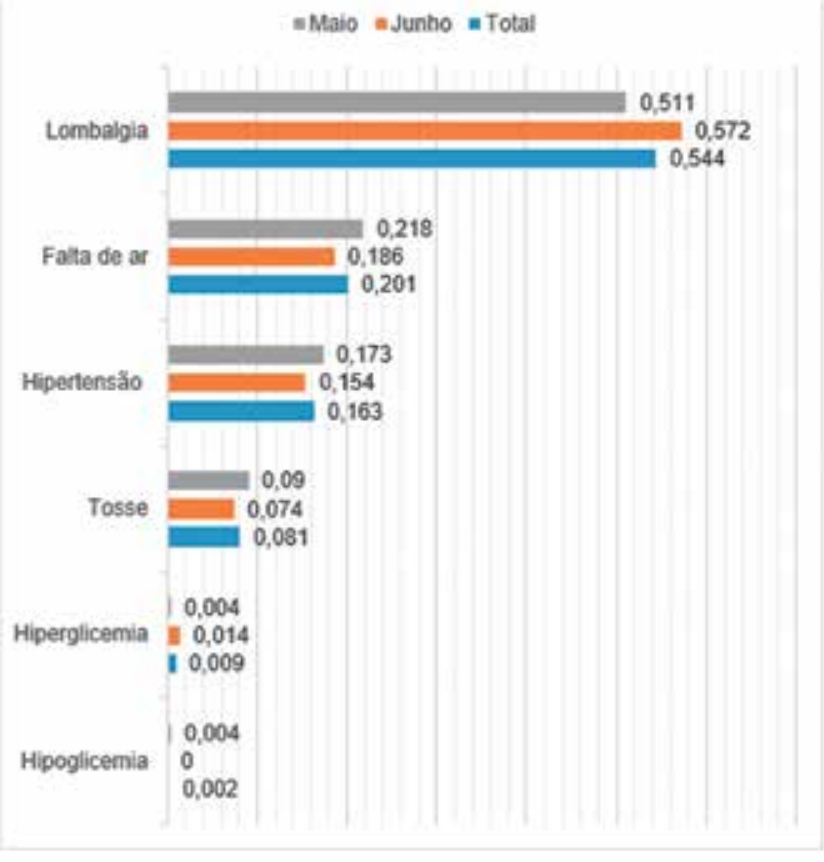

Tabela 4. Análise do decorrer do atendimento. Piracicaba, SP, Brasil, 2018.

\begin{tabular}{|c|c|c|c|c|c|c|}
\hline & \multicolumn{2}{|c|}{ MAIO } & \multicolumn{2}{|c|}{ JUNHO } & \multicolumn{2}{|c|}{ TOTAL } \\
\hline & $\mathrm{N}^{\circ}$ & $\%$ & $\mathrm{~N}^{\circ}$ & $\%$ & $N^{\circ}$ & $\%$ \\
\hline $\begin{array}{l}\text { Queixa = SSVV = } \\
\text { Medicações }\end{array}$ & 187 & $76,9 \%$ & 182 & $63,8 \%$ & 369 & $69,9 \%$ \\
\hline $\begin{array}{l}\text { Queixa = SSVV F } \\
\text { Medicações }\end{array}$ & 0 & 0 & 2 & $0,7 \%$ & 2 & $0,4 \%$ \\
\hline $\begin{array}{l}\text { Queixa } \neq \text { SSVV = } \\
\text { Medicações }\end{array}$ & 13 & $5,4 \%$ & 13 & $4,6 \%$ & 26 & $4,9 \%$ \\
\hline $\begin{array}{l}\text { Queixa = Medica- } \\
\text { ções } \neq \text { SSVV }\end{array}$ & 8 & $3,3 \%$ & 2 & $0,7 \%$ & 10 & $1,9 \%$ \\
\hline $\begin{array}{l}\text { Queixa } \neq \text { SSVV \# } \\
\text { Medicações }\end{array}$ & 3 & $1,2 \%$ & 7 & $2,5 \%$ & 10 & $1,9 \%$ \\
\hline Queixa = SSVV & 21 & $8,7 \%$ & 60 & $21 \%$ & 81 & $15,3 \%$ \\
\hline Queixa $\neq$ SSVV & 11 & $4,5 \%$ & 19 & $6,7 \%$ & 30 & $5,7 \%$ \\
\hline Total & 243 & $100 \%$ & 285 & $100 \%$ & 528 & $100 \%$ \\
\hline DPOC & 11 & $4,5 \%$ & 1 & $0,3 \%$ & 12 & $2,3 \%$ \\
\hline DM & 6 & $2,5 \%$ & 9 & $3,2 \%$ & 15 & $2,8 \%$ \\
\hline HAS + DM & 14 & $5,8 \%$ & 14 & $4,9 \%$ & 28 & $5,3 \%$ \\
\hline HAS & 48 & $19,8 \%$ & 47 & $16,5 \%$ & 95 & $18 \%$ \\
\hline Asma & 48 & $19,8 \%$ & 66 & $23,2 \%$ & 114 & $21,6 \%$ \\
\hline Lombalgia & 107 & $44 \%$ & 137 & $48,1 \%$ & 244 & $46,2 \%$ \\
\hline Total & 243 & $100 \%$ & 285 & $100 \%$ & 528 & $100 \%$ \\
\hline
\end{tabular}

levantados pela Pesquisa Nacional de Saúde (PNS), que foi realizada em 2013 e se repete a cada cinco anos, nela podemos observar que ocorre uma predominância das mulheres na procura por atendimento médico em qualquer nível de atenção, sendo que $77 \%$ das entrevistadas na PNS teriam passado por pelo menos uma consulta médica dentro de doze meses ${ }^{(3)}$.

Diversos estudos mostram que a faixa etária prevalente dos usuários do pronto atendimento, em sua maioria, é de pessoas com a idade de 20 a 29 anos o que difere desta pesquisa. Já no estudo de Feijó e colaboradores ${ }^{(19)}$, a faixa etária prevalente foi de 29 a 59 anos e, nos dados de Guedes e colaboradores $^{(20)}$, a média de idade é de 44 anos e corrobora com os dados colhidos. Autores ressaltam que os atendimentos à faixa etária de 40 a 49 anos apresentam uma inadequação na procura por atendimento nessas unidades ${ }^{(21,22)}$.

Em relação à classificação de risco, as fichas apresentaram uma prevalência da cor verde $(89,2 \%)$. Essa classificação pode ser comparada a outro artigo que apresenta uma demanda de atendimento da cor equivalente ao estudo, como na mostra de Diniz e colabores $^{(23)}$, que foram $61 \%(\mathrm{n}=222)$.

A queixa com maior registro não está classificada pelo Ministério da Saúde como uma DCNT, porém a lombalgia é uma das causas mais frequentes de afastamento do trabalho por incapacidade de realizar as atividades diárias. No estudo, ela apresentou mais da metade dos atendimentos analisados (54,3\%). A pesquisa de Divinópolis, que teve o objetivo de identificar a demanda clínica dos pacientes em uma unidade de pronto atendimento, apresentou $27,1 \%(\mathrm{n}=98)$ dos atendimentos para lombalgia e destaca que essas queixas poderiam ser tratadas na atenção primária a saúde, sendo necessário encaminhar os pacientes para estes serviços e informar aos usuários o funcionamento dos serviços ${ }^{(16,23)}$.

A demanda de atendimentos por dia nas unidades de emergência é alta e pode ocasionar descuidos na triagem dos pacientes, levando a anotações equivocadas na ficha de atendimento. Para que essa si- 


\section{artigo}

Godoi, J.; Campagnoli, M.; Fossa, A.M.; Rocha, M.C.P.; Lino, C.M.; Horibe, T.M.;

Análise da demanda de pacientes com Doenças Crônicas Não Transmissiveis em uma Unidade de Pronto Atendimento:

Gráfico 4. Distribuição dos dados da conduta de acordo com o atendimento médico. Piracicaba, SP, Brasil, 2018.

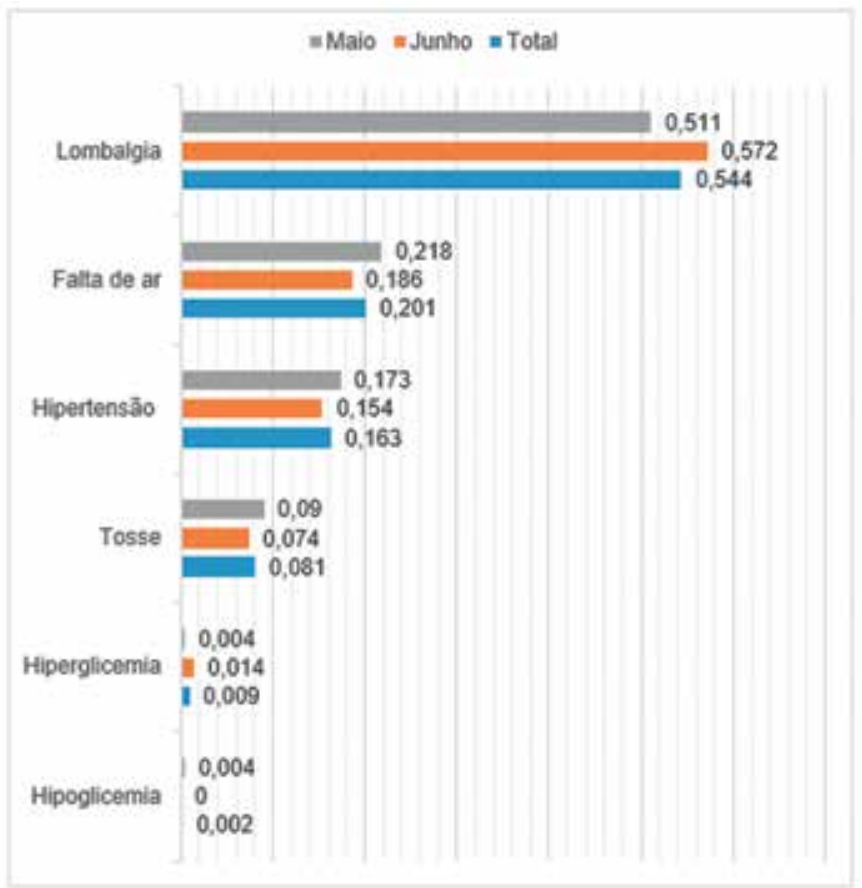

Tabela 5. Distribuição dos dados de acordo com a recidiva dos usuários ao atendimento. Piracicaba, SP, Brasil, 2018
MAIO

\section{Atendimentos \\ mês}

1 à 5

6 à 10

Mais que 10

Total

Queixa $=$ SSVV

Queixa $\neq$ SSVV

Total

DPOC

DM

HAS + DM

HAS

Asma

Lombalgia

Total

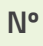

206

22

2

230

21

11

243

11

6

14

48

48

107

243
JUNHO

$\mathrm{N}^{\circ}$

TOTAL

$\begin{array}{lllll}\% & N^{\circ} & \% & N^{\circ} & \%\end{array}$

$89,5 \%$

262

$91,9 \%$

468

$90,9 \%$

$$
9,5 \%
$$

20

$7 \%$

42

$8,1 \%$

$0,8 \%$

3

$1 \%$

5

$1 \%$

$100 \%$

285

$100 \%$

515

$100 \%$

$8,7 \%$

60

$21 \%$

81

$15,3 \%$

$4,5 \%$

19

$6,7 \%$

30

$5,7 \%$

$100 \%$

285

$100 \%$

528

$100 \%$

$4,5 \%$

1

$0,3 \%$

12

$2,3 \%$

$2,5 \%$

$3,2 \%$

15

$2,8 \%$

$5,8 \%$

14

$4,9 \%$

28

$5,3 \%$

$19,8 \%$

47

$16,5 \%$

95

$18 \%$

$19,8 \%$

66

$23,2 \%$

114

$21,6 \%$

$44 \%$

137

$48,1 \%$

244

$46,2 \%$

$100 \%$

$100 \%$

528

$100 \%$ tuação possa ser melhorada, é importante treinar a equipe e escalar pessoas capacitadas para essa função ${ }^{(19)}$.

$\mathrm{Na}$ análise dos dados que relacionam a queixa, os SSVV e as medicações, 69,9\% dos atendimentos são adequados e interligados entre as três etapas, porém é possível observar que algumas FAAs apresentaram discordâncias no decorrer dos atendimentos prestados. A amostra destaca que as queixas no início do atendimento não equivalem aos SSVV e nem às medicações prescritas $(1,9 \%)$, o que pode estar relacionado à falta de informações na ficha, interpretação errada dos profissionais ou discordância no relato do paciente. Outro dado interessante são as queixas equivalentes aos SSVV colhidos na triagem e diferentes das medicações prescritas após $\mathrm{o}$ atendimento médico $(0,4 \%)$.

Ao final do atendimento, é possível observar que houve uma predominância de alta no serviço, sendo o usuário medicado na unidade $(75,6 \%)$ ou liberado com receita médica $(18,9 \%)$, estes dados somam $94 \%$ dos atendimentos. Os artigos $(19,22)$ que tiveram o objetivo de caracterizar a demanda dos atendimentos confirmam uma situação equivalente à da pesquisa, apresentando, respectivamente, $72,7 \%$ e $86,8 \%$, o que representa a maioria dos atendimentos. Os pacientes que foram englobados nesses dados, em sua maioria, eram de classificação verde.

Observou-se que grande parte dos atendimentos era de adultos em idade produtiva, que apresentaram queixas que podem afetar suas atividades diárias e que deveriam ser tratadas a longo prazo pela atenção básica, outras situações de atendimento podem estar relacionadas com a estação do ano em que o estudo foi realizado.

\section{CONCLUSÃO}

No estudo, observamos que a quantidade de atendimentos realizados na UPA relacionados a uma DCNT é baixa em relação aos outros serviços prestados.

Houve predominância do sexo feminino, com a idade de 50 a 54 anos, apresentando uma queixa relacionada às doenças 
crônicas mais comuns no frio, este avaliado que a coleta foi realizada no inverno. As doenças que apresentaram maior incidência na pesquisa foram lombalgia, asma e HAS, que estavam relacionadas às queixas de dor lombar, falta de ar e sintomas de pressão alta, tais quais foram classificadas, em sua maioria, com a cor verde e não apresentavam agravos no quadro do paciente.

É importante ressaltar que algumas fichas não apresentaram concordância entre queixa, SSVV e medicações. Estes dados mostram que as informações nas fichas podem estar incompletas ou que os usuários expressam diferentes queixas a cada profis- sional de saúde, podendo levar a um atendimento ineficaz ao problema existente.

Os desfechos dos atendimentos, em sua maioria, foram de alta após a medicação ou com receita médica. Além disso, observou-se que os pacientes retornam ao serviço de urgência dentro de 30 dias e isto pode estar relacionado à falta de resolução de suas queixas ou à situação apresentada no parágrafo anterior. Outro meio de disseminar essa demanda de pacientes que voltam ao atendimento é realizar o encaminhamento para unidades de referência do seu bairro para início ou continuidade do tratamento de sua DCNT.
$\mathrm{O}$ estudo mostra que não ocorre um encaminhamento para unidades básicas de referência do usuário, o que pode ser a causa do retorno à unidade de emergência. Portanto, é necessário conversar com a equipe sobre a orientação destes usuários quanto à procura das unidades de saúde dos bairros e reforçar com os profissionais a importância de encaminhar estes pacientes, quando necessário. Além disso, a pesquisa mostra altos índices de lombalgias em pacientes com idade produtiva e deve ser um alerta a problemas futuros, como possíveis cirurgias, gerando um custo maior aos serviços de saúde.

\section{REFERÊNCIAS}

1. Ministério da Saúde (BR). Caderno de atenção Básica: Doenças Respiratórias Crônicas. 1. Ed. Brasília: MS; 2010.

2. Ministério da Saúde (BR). Plano de ações estratégicas para o enfrentamento das doenças crônicas não transmissivveis (DCNT) no Brasil 2011-2022. 1. Ed. Brasília: MS; 2011

3. Ministério da Saúde (BR). Diretrizes para o cuidado das pessoas com doenças crônicas nas redes de atenção à saúde e nas linhas de cuidado prioritárias. 1. Ed. Brasília: MS; 2013.

4. Pires MRGM, et al. A Utilização dos Serviços de Atenção Básica e de Urgência no SUS de Belo Horizonte: problema de saúde, procedimentos e escolha dos serviços, Revista Saúde Soc. 2013; 22(1).

5. Amarante LCS. Caracterização da demanda dos serviços de saúde em unidade pronto atendimento segundo critérios de classificação de risco, características sociodemograficas e superutilização. Unicamp; 2014.

6. Gomide MFS, et al. Perfil de usuários em um serviço de pronto atendimento. Revista de Medicina. 2012; 45.

7. Conselho Regional de Enfermagem. Emergências saem do preto e branco. Revista Enfermagem. 2012 nov; 2.

8. Souza CC, Araujo FA, Chianca TCM. Produção científica sobre a validade e confiabilidade do Protocolo de Manchester: revisão integrativa da literatura. Rev esc enferm USP. 2015; 49.

9. Costa JSM. Serviços de Urgência e Emergência hospitalar: atendimento não urgente nas redes de atenção às urgências, um contexto de transformações demográficas. Universidade Federal do Estado de Minas Gerais. Belo Horizonte, MG, 2011.

10. Instituto Brasileiro de Geografia e Estatística. Pesquisa Nacional de Saúde. Percepção do estado de saúde, estilos de vida e doenças crônicas. Brasil, Grandes Regiões e Unidades da Federação. Rio de Janeiro, 2014.

11. Reck PR, et al. Avaliação do equilíbrio postural em trabalhadores com e sem lombalgia. Revista FisiSenectus. 2017 jul/dez, 2.

12. Fonseca JJS. Metodologia da pesquisa científica. Fortaleza:
UEC; 2002.

13. Polit DF, Beck CT, Hungler BP. Fundamentos de pesquisa em enfermagem: métodos, avaliação e utilização. Trad. de Ana Thorell. 5. ed. Porto Alegre: Artmed; 2004.

14. Mattar FN. Pesquisa de marketing. 3.ed. São Paulo: Atlas; 2001.

15. Ministério da Saúde (BR). Manual Instrutivo da Rede de Atenção às Urgências e Emergências no Sistema Único de Saúde (SUS). 1. Ed. Brasília: MS; 2013.

16. Lizier DT, Perez MV, Sakata RK. Exercícios para Tratamento de Lombalgia Inespecífica. Revista Brasileira Anestesiol. 2012; 62.

17. Tomberg JO, et. al. Acolhimento com avaliação e classificação de risco no pronto socorro: caracterização dos atendimentos. Revista Cienc Cuid Saúde. 2013 jan/mar; 12(1).

18. Ribeiro RM, et al. Caracterização do perfil das emergências clínicas no pronto atendimento de um hospital de ensino. REME. 2014 jul/set; 18.

19. Feijó VBER, et al. Análise da demanda atendida em unidade de urgência com classificação de risco. Revista Saúde Debate. 2015 jul/set; 39(106).

20. Guedes HM, et al. Classificação de risco: retrato de população atendida num serviço de urgência brasileiro. Revista de Enfermagem Referência. 2014 fev/mar; IV(1).

21. Garcia VM, Reis RK. Adequação da demanda e perfil de morbidade atendida em uma unidade não hospitalar de urgência e emergência. Revista Cienc Cuid Saúde. 2014 out/dez; 13.

22. Godoi VCG, et al. Acolhimento com classificação de risco: caracterização da demanda em unidade de pronto atendimento. Cogitare Enfermagem. 2016 jul/set; 21.

23. Diniz AS, et al. Demanda clínica de uma unidade de pronto atendimento, segundo o protocolo de Manchester. Rev Eletr Enferm. 2014 abr/jun; 16. 